\title{
The Order of Accuracy for SOR Waveform-Relaxation Method for Solving ODEs
}

Ann J. H. Sawoor

Dept. of Mathematics,

College of Computer Science and Mathematical

University of Mosul, Iraq

Received on: 04/10/2003

Accepted on: 16/12/2003

\section{ABSTRACT}

In this paper we will discuss and find the order of accuracy for subsystems of ODE that obtained from partitioning the successive Over relaxation(SOR) method and we will show that the form of equation to

be solved after applying the waveform relaxation scheme is :

$$
\begin{aligned}
& \dot{x}_{i}^{[k+1]}(t)=f_{i}\left(\omega x_{1}^{[k+1]}, \omega x_{2}^{[k+1]}, \ldots, \omega x_{i-1}^{[k+1]}, x_{i}^{[k+1]},(1-\omega) x_{i}^{[k]}, \omega x_{i+1}^{[k]}, \ldots, \omega x_{m}^{[k]}\right) \\
& x_{i}^{[k+1]}(0)=x_{i, 0} \quad t \in[0, \mathrm{~T}] \quad, 1 \leq i \leq m
\end{aligned}
$$

Where $\omega$, the relaxation parameter should be chosen so that the rate of convergence is maximized .

$$
\dot{x}_{i}^{[k+1]}(t)=f_{i}\left(\omega x_{1}^{[k+1]}, \omega x_{2}^{[k+1]}, \ldots, \omega x_{i-1}^{[k+1]}, x_{i}^{[k+1]},(1-\omega) x_{i}^{[k]}, \omega x_{i+1}{ }^{[k]}, \ldots, \omega x_{m}^{[k]}\right)
$$

Keywords: ordinary differential equation (ODEs), waveform relaxation scheme, order of accuracy, rate of convergence.

$$
\begin{aligned}
& \text { رتبة الدقة للطريقة التذبذبية المسترخية لحل المعادلات التفاضلية الاعتيادية } \\
& \text { آن جلال الساعور } \\
& \text { قسم الرياضيات } \\
& \text { كلية علوم الحاسبات والرياضيات، جامعة الدوصل }
\end{aligned}
$$

$$
\text { تاريخ القبول: 2003/12/16 }
$$

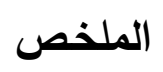

في هذا البحث سوف نناقش ونبين رتبة الدقة للأنظمـة الجزئيـة للمعادلات التفاضلية الاعتيادية الناتجة من عملية تجزئة طريقة (SOR) وسوف نبين أن صيغة المعادلة للحل بعد تطبيق صيغ تذبذبية مسترخية تكون بالثكل الأتي: 


$$
\begin{aligned}
& x_{i}^{[k+1]}(0)=x_{i, 0} \quad t \in[0, \mathrm{~T}] \quad, 1 \leq i \leq m \\
& \text { حيث } \omega \text { تمثل معلمـة للاسـترخاء والتي يجب أن تختـار بحيث أن نسبة } \\
& \text { التقارب عند هذه القيمة للمعلمة تكون في أعظمها. }
\end{aligned}
$$

الكلمـات المفتاحيـة: المعادلات التفاضلية الاعتيادية، الطريقة التذبذبية المسترخية،

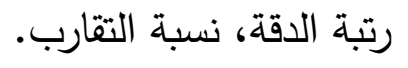

\section{1- Introduction:}

Waveform method were first proposed ([1],[2],[3]\&[4]) in the context VLSI circuit simulation where they were used to solve differential-algebraic (DAEs) . We will examine in this paper, the SOR waveform relaxation effectiveness for the solution of ordinary differential equation (ODEs) which is special case of DAEs. A waveform is a continuous representation of a solution component on a window.

Consider the following system of ordinary differential equations:

$$
\frac{\mathrm{dx}}{\mathrm{dt}}=\mathrm{F}(\mathrm{t}, \mathrm{x}) \quad \text { or } \quad \dot{x}=F(x) \quad, \quad x(0)=x_{0}
$$

where $x \in R^{n}$ and $F: R^{n} \rightarrow R^{n}$. Using waveform relaxation to solve (1), the system is first partitioned into $\mathrm{m}$ coupled subsystems:

$$
\begin{aligned}
& \dot{x}_{1}=f_{1}\left(x_{1}, x_{2}, \ldots, x_{m}\right) \quad, \quad x_{1}(0)=x_{1,0} \\
& \vdots \\
& \dot{x}_{m}=f_{m}\left(x_{1}, x_{2}, \ldots, x_{m}\right) \quad, \quad x_{m}(0)=x_{m, 0}
\end{aligned}
$$

where 


$$
\begin{gathered}
x_{i} \in R^{n i}, x=\left(x_{1}{ }^{T}, x_{2}{ }^{T}, \ldots, x_{m}{ }^{T}\right)^{T}, \quad f_{i}: R^{n} \rightarrow R^{n i}, F=\left(f_{1}^{T}, f_{2}{ }^{T}, \ldots, f_{m}{ }^{T}\right)^{T} \\
, 1 \leq i \leq m \text { and } \sum_{i=1}^{m} n_{i}=n
\end{gathered}
$$

$x_{1}^{T}, x_{2}{ }^{T}, \ldots, x_{m}{ }^{T}$ are vectors in $\mathrm{R}^{\mathrm{n}}$. Where

then each subsystem :

$$
\dot{x}_{i}=f_{i}\left(x_{1}, x_{2}, \ldots, x_{i-1}, x_{i}, x_{i+1}, \ldots, x_{m}\right) \quad, x_{i}(0)=x_{i, 0}, 1 \leq i \leq m
$$

is solved independently by using past values of $\mathrm{x}_{1}, \mathrm{x}_{2}, \ldots, \mathrm{x}_{\mathrm{i}-}$

${ }_{1}, \mathrm{X}_{\mathrm{i}+1}, \ldots, \mathrm{X}_{\mathrm{m}}$.

\section{Lemma (1):}

In successive Over-Relaxation (SOR) method, consider the equation for the ith component after partitioning

$$
\dot{x}_{i}=f_{i}\left(x_{1}, x_{2}, \ldots, x_{i}, \ldots, x_{m}\right) \quad, x_{i}(0)=x_{i, 0}, 1 \leq i \leq m
$$

Then the equation to be solved after applying the waveform relaxation scheme is :

$$
\begin{aligned}
& \dot{x}_{i}^{[k+1]}(t)=f_{i}\left(\omega x_{1}^{[k+1]}, \omega x_{2}^{[k+1]}, \ldots, \omega x_{i-1}^{[k+1]}, x_{i}^{[k+1]},(1-\omega) x_{i}^{[k]}, \omega x_{i+1}^{[k]}, \ldots, \omega x_{m}^{[k]}\right) \ldots . \\
& x_{i}^{[k+1]}(0)=x_{i, 0} \quad t \in[0, T] \quad, 1 \leq i \leq m
\end{aligned}
$$

Where $\omega$ is the relaxation parameter which should be chosen so that the rate of convergence is maximized. Also, if $\omega=1$, then eq. (2) will be reduced to waveform Gauss-Seidel method.

\section{Proof:}

Consider the SOR iterative formula:

$$
x_{i}^{[k+1]}=(1-\omega) x_{i}^{[k]}+\frac{\omega}{a_{i i}}\left[b_{i}-\sum_{j=1}^{i-1} a_{i j} x_{j}^{[k+1]}-\sum_{j=i+1}^{m} a_{i j} x_{j}^{[k]}\right]
$$

now multiplying both sides of (3) by $\mathrm{a}_{\mathrm{ii}}$ and collect all $[\mathrm{k}+1]$ th iterate term to give :

$$
a_{i i} x_{i}^{[k+1]}=(1-\omega) a_{i i} x_{i}^{[k]}+\omega\left[b_{i}-\sum_{j=1}^{i-1} a_{i j} x_{j}^{[k+1]}-\sum_{j=i+1}^{m} a_{i j} x_{j}^{[k]}\right]
$$




$$
\begin{aligned}
& a_{i i} x_{i}^{[k+1]}+\omega \sum_{j=1}^{i-1} a_{i j} x_{j}^{[k+1]}=(1-\omega) a_{i i} x_{i}^{[k]}-\omega \sum_{j=i+1}^{m} a_{i j} x_{j}^{[k]}+\omega b_{i} \\
& \begin{aligned}
\omega\left(a_{i 1} x_{1}^{[k+1]}+a_{i 2} x_{2}^{[k+1]}+\ldots+a_{i, i-1} x_{i-1}{ }^{[k+1]}\right)+a_{i i} x_{i}^{[k+1]}= \\
(1-\omega) a_{i i} x_{i}^{[k]}-\omega\left(a_{i, i+1} x_{i}^{[k]}+\ldots+a_{i m} x_{m}{ }^{[k]}\right)+\omega b_{i}
\end{aligned} \\
& \begin{array}{c}
\omega a_{i 1} x_{1}^{[k+1]}+\omega a_{i 2} x_{2}^{[k+1]}+\ldots+\omega a_{i, i-1} x_{i-1}{ }^{[k+1]}+a_{i i} x_{i}^{[k+1]}= \\
(1-\omega) a_{i i} x_{i}^{[k]}-\omega a_{i, i+1} x_{i}^{[k]}-\ldots-\omega a_{i m} x^{[k]}+\omega b_{i}
\end{array}
\end{aligned}
$$

Now since SOR method solved $A x=b$, so we have :

$$
x_{i}^{[k+1]}=\left(\omega x_{1}^{[k+1]}, \omega x_{2}^{[k+1]}, \ldots, \omega x_{i-1}^{[k+1]}, x_{i}^{[k+1]},(1-\omega) x_{i}^{[k]}, \omega x_{i+1}{ }^{[k]}, \ldots, \omega x_{m}{ }^{[k]}\right)^{T}
$$

Also consider the following autonomous system of ODEs

$$
\dot{x}=F(x) \quad, x(0)=x_{0}
$$

Where $x \in R^{n}$ and $F: R^{n} \rightarrow R^{n}$.. Since this system is partitioned into $\mathrm{m}$ coupled subsystems

$$
\dot{x}_{i}=f_{i}\left(x_{i}\right) \quad, x_{i}(0)=x_{i, 0} \quad 1 \leq i \leq m
$$

Thus we obtain

$$
\begin{aligned}
& \dot{x}_{i}^{[k+1]}=f_{i}\left(x_{i}^{[k+1]}\right) \\
& \dot{x}_{i}^{[k+1]}(t)=f_{i}\left(\omega x_{1}^{[k+1]}, \omega x_{2}^{[k+1]}, \ldots, \omega x_{i-1}^{[k+1]}, x_{i}^{[k+1]},(1-\omega) x_{i}^{[k]}, \omega x_{i+1}{ }^{[k]}, \ldots, \omega x_{m}{ }^{[k]}\right) \ldots . \\
& x_{i}^{[k+1]}(0)=x_{i, 0} \quad t \in[0, T] \quad, 1 \leq i \leq m
\end{aligned}
$$

Finally if $\omega=1$ in (4) we get the equation (5) bellow that can be solved by waveform Gauss-Seidel method.

$$
\begin{aligned}
& \dot{x}_{i}^{[k+1]}(t)=f_{i}\left(x_{1}^{[k+1]}, x_{2}^{[k+1]}, \ldots, x_{i-1}^{[k+1]}, x_{i}^{[k+1]}, x_{i+1}{ }^{[k]}, \ldots, x_{m}{ }^{[k]}\right) \\
& x_{i}^{[k+1]}(0)=x_{i, 0} \quad, t \in[0, T] \quad, 1 \leq i \leq m
\end{aligned}
$$

Hence the proof is complete. \# 


\section{2-Accuracy for subsystem:}

In this section, instead of discussing the convergence property of waveform relaxation,we will discuss the order of accuracy for subsystem of ODEs that is obtained from partitioning the SOR method because all waveform relaxation methods converge superlinearly on any finite inte-rval so it is not possible to use as a measure like the order of accuracy. Thus we will look at waveform relaxation from a different point of view. This will be shown in the following theorem:

\section{Theorem (1):}

In SOR method, consider the equation for the ith component after partitioning,

$$
\dot{x}_{i}=f_{i}\left(x_{1}, x_{2}, \ldots, x_{i}, \ldots, x_{m}\right), x_{i}(0)=x_{i, 0}, 1 \leq i \leq m
$$

The equation to be solved after applying the waveform relaxation scheme is:

$$
\begin{aligned}
& \dot{x}_{i}^{[k+1]}(t)=f_{i}\left(\omega x_{1}^{[k+1]}, \omega x_{2}^{[k+1]}, \ldots, \omega x_{i-1}^{[k+1]}, x_{i}^{[k+1]},(1-\omega) x_{i}^{[k]}, \omega x_{i+1}^{[k]}, \ldots, \omega x_{m}^{[k]}\right) \ldots(7) \\
& x_{i}^{[k+1]}(0)=x_{i, 0} \quad t \in[0, \mathrm{~T}] \quad, 1 \leq i \leq m
\end{aligned}
$$

Assume that

$$
\begin{array}{ll}
E_{j}^{[K+1]}=x_{j}^{[k+1]}-x_{j}=O(t)^{N_{j}^{[k+1]}} & \text { for } j \leq i \\
E_{j}^{[K]}=x_{j}^{[k]}-x_{j}=O(t)^{N_{j}^{[k]}} & \text { for } j \succ i
\end{array}
$$

And all the $E_{j}^{[k]} s \& E_{j}^{[K+1]} s$ are sufficiently smooth. Then the order of accuracy denoted by $\mathrm{N}$ is given by: $N_{i}^{[k+1]} \geq \min \left(\omega N_{1}^{[k+1]}, \ldots, \omega N_{i-1}{ }^{[k+1]},(1-\omega) N_{i}^{[k]}, \omega N_{i+1}{ }^{[k]}, \ldots, \omega N_{m}{ }^{[k]}\right)+1 \ldots \ldots .(9)$ with equality unless there is a numerical cancellation . 


\section{Proof:}

According to lemma(1), we only need to prove that the order of accuracy noted by $\mathrm{N}$ is given by eq.(9).

Let

$$
M=\min \left(\omega N_{1}^{[k+1]}, \ldots, \omega N_{i-1}^{[k+1]},(1-\omega) N_{i}^{[k]}, \omega N_{i+1}^{[k]}, \ldots, \omega N_{m}{ }^{[k]}\right),
$$

Then for $\mathrm{r}=0,1, \ldots, \mathrm{M}-1$

$\frac{d^{r}}{d t^{r}} E_{j}^{[k+1]}(0)=0 \quad$ for $j=1,2, \ldots, i-1$,

$\frac{d^{r}}{d t^{r}} E_{j}^{[k]}(0)=0 \quad$ for $j=i+1, \ldots, m$

From (6)\&(7) we have

$$
\begin{aligned}
\dot{E}_{i}^{[k+1]}= & f_{i}\left(\omega x_{1}^{[k+1]}, \ldots, \omega x_{i-1}^{[k+1]}, x_{i}^{[k+1]},(1-\omega) x_{i}^{[k]}, \omega x_{i+1}{ }^{[k]}, \ldots, \omega x_{m}{ }^{[k]}\right)- \\
& -f_{i}\left(x_{1}, x_{2}, \ldots, x_{i}, \ldots, x_{m}\right) \\
= & \omega \sum_{j<i} f_{i, j} E_{j}^{[K+1]}+f_{i, i} E_{i}^{[K+1]}+(1-\omega) f_{i, i} E_{i}^{[k]}+\sum_{j>i} f_{i, j} E_{j}^{[k]} \ldots \ldots \ldots . . .(1)
\end{aligned}
$$

By (7)\&(10) we see that $\dot{E}_{i}^{[k+1]}(0)=0$ from (11).

Now differentiate (11) w.r.t. $t$ to get :

$$
\begin{aligned}
\ddot{E}_{i}^{[k+1]} & =\left\{\frac{d}{d t} f_{i, i} E_{i}^{[k+1]}+f_{i, i} \dot{E}_{i}^{[k+1]}\right\}+(1-\omega)\left\{\frac{d}{d t} f_{i, i} E_{i}^{[K]}+f_{i, i} \dot{E}_{i}^{[K]}\right\}+ \\
& +\omega \sum_{j<i}\left\{\frac{d}{d t} f_{i, j} E_{j}^{[k+1]}+f_{i, i} \dot{E}_{j}^{[k+1]}\right\}+\omega \sum_{j \succ i}\left\{\frac{d}{d t} f_{i, j} E_{j}^{[k]}+f_{i, i} \dot{E}_{j}^{[k]}\right\}
\end{aligned}
$$

Since

$$
\begin{aligned}
& \dot{E}_{j}^{[K+1]}(0)=E_{j}^{[k+1]}(0)=0 \quad \text { for } j \leq i \\
& \dot{E}_{j}^{[K]}(0)=E_{j}^{[K]}(0)=0 \quad \text { for } j \succ i
\end{aligned}
$$

From $(8) \& \dot{E}_{i}^{[K+1]}(0)=0 \quad$ we have $\ddot{E}_{i}^{[K+1]}(0)=0$

Differentiate equation (11) $\mathrm{r}$ times, we get the following general form: 


$$
\begin{aligned}
\frac{d^{r+1}}{d t^{r+1}} E_{i}^{[K+1]}(t)= & \sum_{l=0}^{r}\left(\begin{array}{l}
r \\
l
\end{array}\right) \frac{d^{l}}{d t^{l}} f_{i, i}(t) \frac{d^{r-l}}{d t^{r-l}} E_{j}^{[k+1]}(t)+ \\
& +(1-\omega) \sum_{l=0}^{r}\left(\begin{array}{l}
r \\
l
\end{array}\right) \frac{d^{l}}{d t^{l}} f_{i, i}(t) \frac{d^{r-l}}{d t^{r-l}} E_{j}^{[k]}(t)+ \\
& +\omega \sum_{j<i} \sum_{l=0}^{r}\left(\begin{array}{l}
r \\
l
\end{array}\right) \frac{d^{l}}{d t^{l}} f_{i, j}(t) \frac{d^{r-l}}{d t^{r-l}} E_{j}^{[k+1]}(t)+ \\
& +\omega \sum_{j \succ i} \sum_{l=0}^{r}\left(\begin{array}{l}
r \\
l
\end{array}\right) \frac{d^{l}}{d t^{l}} f_{i, j}(t) \frac{d^{r-l}}{d t^{r-l}} E_{j}^{[k]}(t) \ldots \ldots \ldots . . .
\end{aligned}
$$

By induction we get

$\frac{d^{M}}{d t^{M}} E_{i}^{[k+1]}(0)=0$.

$\frac{d^{M+1}}{d t^{M+1}} E_{i}^{[k+1]}(0) \quad$ will not be zero if there exists some $j \neq i$ such that :

$\frac{d^{M}}{d t^{M}} E_{j}^{[k+1]}(0) \neq 0 \quad$ for some $j \prec i$

or

$\frac{d^{M}}{d t^{M}} E_{j}^{[k]}(0) \neq 0 \quad$ for some $j \succ i$

with the corresponding $f_{i, j} \neq 0$, unless there is a numerical cancellation.

\section{Particular Case:}

To illustrate Theorem (1) we can take a particular case as follows:

In particular, we consider a system of three equations (which can be considered as the general case of any example consists of a system of three equations): 


$$
\begin{aligned}
& \dot{x}_{1}{ }^{[k+1]}=f_{1}\left(\omega x_{1}{ }^{[k+1]}, \omega x_{2}{ }^{[k]}, x_{3}{ }^{[k]},(1-\omega) x_{3}{ }^{[k]}\right) \\
& \dot{x}_{2}{ }^{[k+1]}=f_{2}\left(\omega x_{1}{ }^{[k+1]}, \omega x_{2}{ }^{[k+1]}, x_{3}{ }^{[k]},(1-\omega) x_{3}{ }^{[k]}\right) \\
& \dot{x}_{3}{ }^{[k+1]}=f_{3}\left(\omega x_{1}{ }^{[k+1]}, \omega x_{2}{ }^{[k+1]}, x_{3}{ }^{[k+1]},(1-\omega) x_{3}{ }^{[k]}\right) \\
& x_{1}{ }^{[k]}-x_{1}=O(t)^{N_{1}{ }^{[k]}} \\
& x_{2}{ }^{[k]}-x_{2}=O(t)^{N_{2}{ }^{[k]}} \\
& x_{3}{ }^{[k]}-x_{3}=O(t)^{N_{3}{ }^{[k]}}
\end{aligned}
$$

Then from the previous theorem we have:

$$
\begin{aligned}
& N_{1}^{[K+1]} \geq \min \left(N_{2}{ }^{[K]}, N_{3}{ }^{[K]}\right)+1 \\
& N_{2}{ }^{[K+1]} \geq \min \left(N_{1}{ }^{[K+1]}, N_{3}{ }^{[K]}\right)+1 \\
& N_{3}{ }^{[K+1]} \geq \min \left(N_{1}{ }^{[K+1]}, N_{2}{ }^{[K+1]}\right)+1
\end{aligned}
$$

This theorem assumes that all variables appear in all equations. If variable $\mathrm{j}$ appears in the equation for variable $\mathrm{i}$ only if $j \in I_{i}$ where $\mathrm{I}_{\mathrm{i}}$ is a subset of $[1,2,3]$, then equation (9) can be replaced by:

$$
N_{i}^{[K+1]} \geq \min _{j \in I_{i}}\left(N_{j}^{[K+H(i-j)]}\right)+1
$$

Where $\mathrm{H}(\mathrm{i}-\mathrm{j})=1$ if $i \succ j$ and 0 otherwise.

\section{3-Conclusion:}

In this paper we discussed the accuracy increase property for a special approach, the SOR waveform relaxation method. We obtained that the accuracy increase after one sweep of SOR waveform relaxation is usually greater than one. 


\section{REFERENCES}

[1] Juang, F. L., 1990, Waveform methods for ordinary differential equations, Report No. UIUCDCS-R-90-1563. UILU-ENG-90-1701, DOE/ER /25026/34.

[2] Wells, D. R. , 1982, Multirate linear multistep methods for the solution of systems of ordinary differential equations, Ph.D. Thesis, university of Illinois at Urbana-Champaign. U.S.A.

[3] Wells, D. R. \& Gear, C.W., 1985, Multirate linear multistep methods, University of Illinois at Urbana-Champaign. U.S.A.

[4] Zbigniew Bartoszewski, 2000, Numerical verification of delay dependent error estimates for Waveform Relaxation Method (WRM) for differential -functional equations. $9^{\text {th }}$ seminar on numerical solution of differential and differential algebraic equations. NUMDIFF-9, 4 September - 8 September 2000, Halle (Germany). 